\title{
The Capsules of Corynebacterium equi and Streptococcus equi
}

\author{
By J. B. WOOLCOCK AND M. D. MUTIMER \\ Department of Veterinary Pathology and Public Health, \\ University of Queensland, St Lucia, Brisbane, Australia
}

(Received 3 May 1978; revised 27 June 1978)

The capsules of Corynebacterium equi and Streptococcus equi were examined by electron microscopy after staining with ruthenium red. They were compared with the capsule of Klebsiella pneumoniae which had previously been examined using the same procedure (Springer \& Roth, 1973). The capsule of C. equi had a laminated appearance. When S. equi was grown on solid medium, its capsule appeared as radially arranged projections capped by a thick electron dense layer. When grown in liquid medium, $S$. equi produced a capsule which showed as short thick projections with no layer external to them.

\section{INTRODUCTION}

The ultrastructure and chemical composition of the capsules of many Gram-negative bacteria have been studied (Cagle, 1975; Sutherland, 1972). However, apart from the streptococcal polysaccharides and the dextran producers, very little detailed information has been published relating to Gram-positive organisms (Stacey \& Barker, 1960; Sutherland, 1972). The structure of a bacterium has significance in terms of the role(s) which each component plays in the pathogenesis of the particular infection. Corynebacterium equi is associated with a severe bronchopneumonia in foals and grows as a mucoid colony with capsules that can be demonstrated by standard procedures. This paper compares the ultrastructural appearance of the capsule of C. equi with the capsules of Streptococcus equi and Klebsiella pneumoniae whose composition is known.

\section{METHODS}

Strains and media. Corynebacterium equi uQv1050 was freshly isolated from the lung of a foal with bronchopneumonia. It was maintained on peptone/yeast extract agar slopes. Streptococcus equi UQv138 was recovered from an equine submandibular lymph node abscess, and was maintained on slopes of blood agar base (Difco) supplemented with $5 \%(\mathrm{v} / \mathrm{v})$ sheep blood. Klebsiella pneumoniae uQv60, of animal origin, was reconstituted from the freeze-dried state and maintained on peptone/yeast extract agar slopes.

Corynebacterium equi and $K$. pneumoniae were grown overnight in peptone/yeast extract broth at $37^{\circ} \mathrm{C}$ : $C$. equi was incubated as a shaken culture, while $K$. pneumoniae was grown under static conditions. Streptococcus equi was grown for $13 \mathrm{~h}$ in Todd-Hewitt broth (Oxoid) supplemented with $1 \%(\mathrm{w} / \mathrm{v}$ ) glucose and this culture was used to inoculate fresh medium which was incubated for $4 \mathrm{~h}$ with occasional shaking. Streptococcus equi and C. equi were also grown on sheep blood agar overnight.

Electron microscopy. All liquid cultures were centrifuged and the deposited cells were stained and fixed by the method of Springer \& Roth (1973). Streptococcus equi grown on solid medium was washed off the plates using phosphate-buffered saline and then treated in the same way as the broth cultures. Dehydration and embedding were done by Luft's method as described by Springer \& Roth (1973). All sections were examined with an AEI Corinth type 275 electron microscope. 

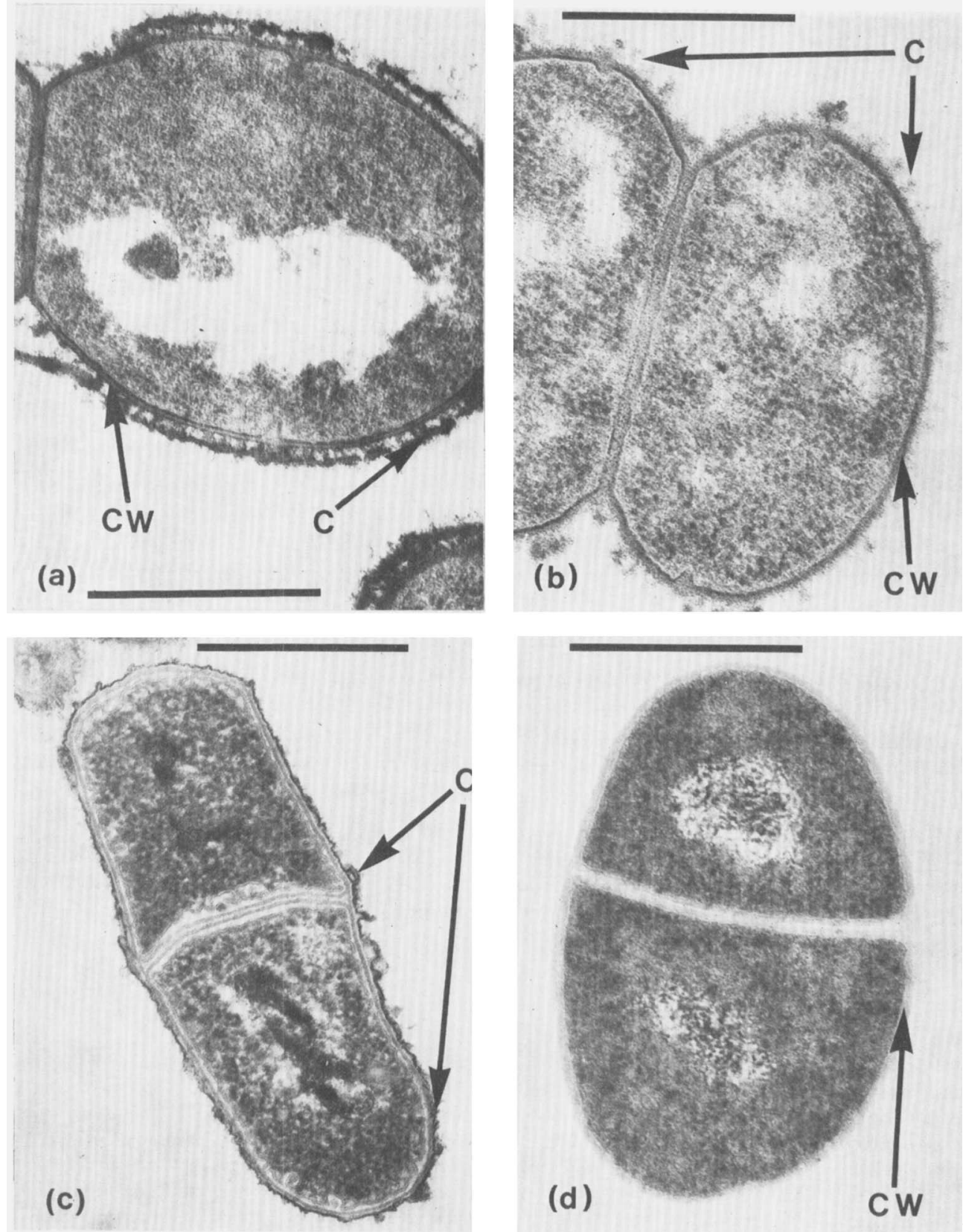

Fig. 1. (a) Streptococcus equi from growth on solid medium: the capsular material (C) stains with ruthenium red and appears as radial projections from the cell wall (CW) capped by a thick electron dense layer. (b) Streptococcus equi from growth in liquid medium: C shows only as short thick projections from $\mathrm{CW}$. (c) Corynebacterium equi showing the laminated appearance of $\mathrm{C}$ stained with ruthenium red. $(d)$ Corynebacterium equi, stainless control: no capsular material is evident external to $\mathrm{CW}$. This organism is quite pleomorphic, which accounts for the differing morphology compared with $(c)$. Bar markers represent $0.5 \mu \mathrm{m}$. 


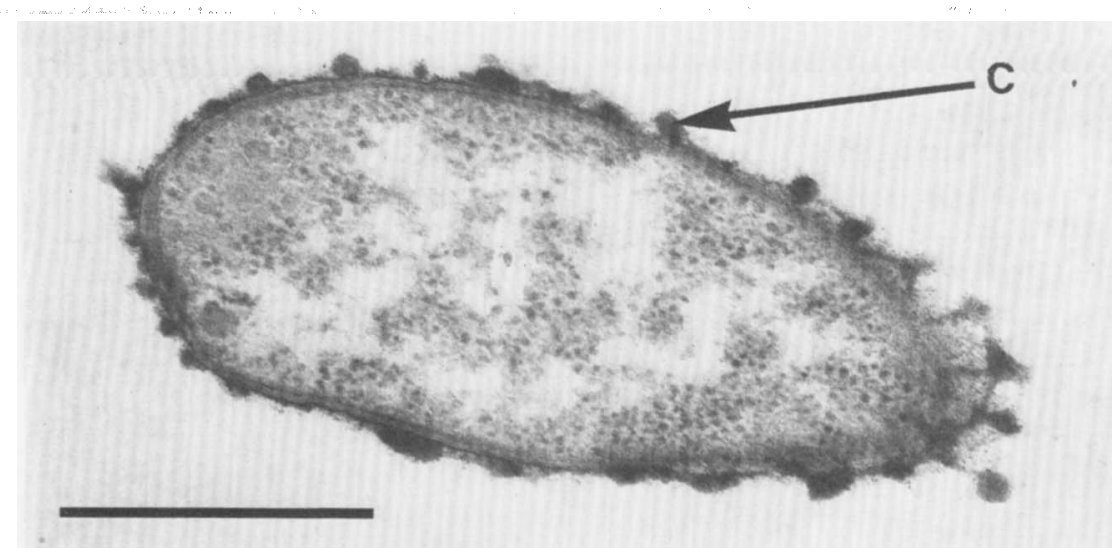

Fig. 2. Klebsiella pneumoniae with the capsular material (C) showing as globular arrangements around the cell. Bar marker represents $0.5 \mu \mathrm{m}$.

\section{RESULTS}

\section{Streptococcus equi}

The wall, typical of Gram-positive bacteria, consisted of two electron dense layers separated by a less dense zone. The appearance of the capsule which stained with ruthenium red depended on whether the organisms derived from liquid or solid media. When $S$. equi was grown on agar, the surface coat consisted of radially arranged projections capped by a thick electron dense layer (Fig. 1a). Occasional organisms were seen in which the coat appeared as an uneven electron dense layer without projections. When grown in a liquid medium, the outer covering of $S$. equi had a different appearance (Fig. 1b). Outside the wall only short thick projections were seen, with no layer external to them. These projections in no way resembled the surface fimbriae which have been demonstrated in group A streptococci (Swanson et al., 1969). When ruthenium red was not used for staining, no structures were visible external to the wall.

\section{Corynebacterium equi}

The capsule of $C$. equi showed a further variation in appearance. Staining with ruthenium red occurred, but there was no suggestion of any projecting structures. Instead, the capsule had a wavy appearance and seemed to be almost laminated (Fig. 1c). In some preparations up to three layers were apparently present. The appearance of the capsular material was similar whether the organism was grown on solid or in liquid medium. No such layers could be seen in organisms not stained by ruthenium red (Fig. 1d).

\section{Klebsiella pneumoniae}

Capsular material was seen as globular arrangements around the cells (Fig. 2). These were not unlike the short projections of $S$. equi but were denser, thicker and more rounded.

\section{DISCUSSION}

The purpose of this study was to establish whether the capsule of $C$. equi could be stained and visualized using ruthenium red. This dye in combination with fixation with osmium tetroxide is able to stain polysaccharides (Luft, 1971). The procedure has been utilized to examine bacterial capsules by electron microscopy since binding between the dye and the capsule enhances visualization (Cagle et al., 1972; Springer \& Roth, 1973; Brooker, 1976). Such ultrastructural studies have been performed on both Gram-positive and Gram- 
negative bacteria, and they indicate a variety of arrangements of the capsular polymers. It is clear from the present study that the many variations which can occur in both organisms and technical procedures markedly affect the appearance of capsules examined in the dehydrated state by electron microscopy.

We included $S$. equi in our study so that we could compare C. equi with an organism whose capsular composition and production is known. Structurally the capsules of these two organisms are quite different. Indeed the appearance of the $S$. equi capsule differs according to cultural conditions. On solid media, where growth occurs as mucoid, sticky colonies, individual organisms presumably synthesize hyaluronic acid which stays largely attached to the wall. This higher proportion of solid to liquid in the capsule of such organisms could account for the greater concentration of ruthenium red staining material seen by electron microscopy. On the other hand, when $S$. equi grows in liquid medium, there is copious release of hyaluronic acid into the medium (Woolcock, 1974), thus lowering the ratio of solid to liquid in the capsule and consequently leaving less polymer available for staining.

Klebsiella pneumoniae was included in this study as the ultrastructure of its capsule has been examined by the same procedures as were used here (Springer \& Roth, 1973). We were not able to demonstrate the capsular fibrils as described, but the globular appearance of the capsule in our preparations has been illustrated by these authors.

Both Springer \& Roth (1973) and Cagle (1975) give details of the dimensions of capsular fibrils observed by them. They also look for significance in the differing arrangements of the capsular polymers. Such discussion would appear to have limited value since the capsule is mostly water with a small fraction of solid material and preparation for electron microscopy involves dehydration, when distortion from the natural state must occur. Admittedly the fibrillar visualization of the capsule is in keeping with our conception of the capsule in chemical terms, i.e. thread-like polymers. However, whether these threads appear as globules, spikes or fibrils must largely be determined by factors involved in growth of the organisms and in preparation of the specimens. For this reason we prefer not to speculate on the significance of the ruthenium red staining laminations of $C$. equi. Variations in arrangement of capsular polymers are of interest to the bacterial morphologist, but such observations do not help to determine the role which the capsule may play in the pathogenesis of the infection with which it is associated.

This study was supported in part by a grant from the Queensland Equine Research Foundation.

\section{REFERENCES}

BROOKER, B. E. (1976). Surface coat transformation and capsule formation by Leuconostoc mesenteroides NCDO 523 in the presence of sucrose. Archives of Microbiology 111, 99-104.

Cagle, G. D. (1975). Fine structure and distribution of extracellular polymer surrounding selected aerobic bacteria. Canadian Journal of Microbiology 21, 395-408.

Cagle, G. D., Pfister, R. M. \& Vela, G. R. (1972). Improved staining of extracellular polymer for electron microscopy: examination of Azotobacter, Zoogloea, Leuconostoc and Bacillus. Applied Microbiology 24, 477-487.

LuFT, J. H. (1971). Ruthenium red and violet I. Chemistry, purification, methods of use for electron microscopy and mechanism of action. Anatomical Record 171, 347-368.

SPRINGER, E. L. \& Roth, 1. L. (1973). The ultra- structure of the capsules of Diplococcus pneumoniae and Klebsiolla pneumoniae stained with ruthenium red. Journal of General Microbiology 74, 21-31.

StaCey, M. \& Barker, S. A. (1960). Polysaccharides of Microorganisms. Oxford: Oxford University Press.

Sutherland, I. W. (1972). Bacterial exopolysaccharides. Advances in Microbial Physiology 8, 143-213.

Swanson, J., Hsu, K. C. \& Gotschlich, E. C. (1969). Electron microscopic studies on streptococci I.M antigen. Journal of Experimental Medicine 130, 1063-1091.

Woolcock, J. B. (1974). The capsule of Streptococcus equi. Journal of General Microbiology 85, 372-375. 